PROCEEDINGS OF THE

AMERICAN MATHEMATICAL SOCIETY

Volume 45, Number 2, August 1974

\title{
AN EXTENSION OF THE HAUSDORFF-YOUNG THEOREM
}

\author{
ROBERT M. YOUNG
}

ABSTRACT. Using the Riesz-Thorin interpolation theorem, we show that if $1<p<2$ and $f$ belongs to $L^{p}(-\pi, \pi)$, then $\left\{\hat{f}\left(z_{n}\right)\right\}$ belongs to $l^{q}(q=p /(p-1))$ for a very general class of complex sequences $\left\{z_{n}\right\}_{0}$ We also obtain a convergence criterion for a related class of exponential sums.

1. Introduction. The classical Hausdorff-Young theorem states that if $1<p<2$ and $f$ belongs to $L^{p}(-\pi, \pi)$, then $\{\hat{f}(n)\}$ belongs to $l^{q}$, where $q$ is the conjugate exponent, that is $q=p /(p-1)$. In this note we offer a simple proof showing that $\left\{\hat{f}\left(z_{n}\right)\right\}$ belongs to $l^{q}$ for a very general class of complex sequences $\left\{z_{n}\right\}$. We also obtain a convergence criterion for a related class of exponential sums. Our results, although seemingly known, do not appear to be in the literature.

Definition. A sequence $\left\{z_{n}\right\}$ of distinct complex numbers will be called separated if there is a constant $\delta>0$ such that $\left|z_{n}-z_{m}\right| \geq \delta$ for all $n \neq m$.

Theorem. Let $\left\{z_{n}\right\}$ be a separated sequence of points lying in a strip parallel to the real axis. Let $1<p<2$ and let $q$ be the conjugate exponent.

(i) There is a constant $A$ such that the inequality

$$
\left\|\sum c_{n} e^{i z n}\right\|_{q} \leq A\left(\sum\left|c_{n}\right|^{p}\right)^{1 / p}
$$

holds whenever $\left\{c_{n}\right\}$ belongs to $l^{p}$.

(ii) If $f \in L^{p}(-\pi, \pi)$, then

$$
\left(\sum\left|\hat{f}\left(z_{n}\right)\right|^{q}\right)^{1 / q} \leq A\|f\|_{p}
$$

with $A$ as above.

2. Preliminary lemma. The following lemma was proved by Titchmarsh [4] for the case when $z_{n}$ is real and later reproved by Paley and Weiner [3] and Ingham [1]. The proof in the general case is a simple extension of the argument given in [1] and is therefore omitted.

Received by the editors September 1, 1972.

AMS (MOS) subject classifications (1970). Primary 42A16; Secondary 40 A05.

Key words and phrases. Exponential sums, separated sequence, almost periodic functions, interpolation of operators. 
Lemma. Let $\left\{z_{n}\right\}$ be a separated sequence of points lying in a strip parallel to the real axis. There is a constant $A$ such that

$$
\frac{1}{2 \pi} \int_{-\pi}^{\pi}\left|\sum c_{n} e^{i z n^{t}}\right|^{2} d t \leq A \sum\left|c_{n}\right|^{2}
$$

whenever $\Sigma\left|c_{n}\right|^{2}<\infty$.

3. Proof of the theorem. If $\left\{c_{n}\right\} \in l^{1}$, then

$$
\left|\sum c_{n} e^{i z n^{t}}\right| \leq A \sum\left|c_{n}\right|
$$

for some absolute constant $A$. The inequalities (3), (4) show that the mapping $T: l^{2} \rightarrow L^{2}$ given by $\left\{c_{n}\right\} \mapsto \Sigma_{n} e^{i z_{n} t}$ is continuous and that the restriction mapping $T: l^{1} \rightarrow L^{\infty}$ is also continuous. It follows from the RieszThorin theorem [2, p. 97] that the restriction of $T$ to $l^{p}$ is a bounded operator from $l^{p}$ into $L^{q}$, and this establishes (1).

Now inequality (2) follows immediately from (1) since we can choose complex numbers $d_{n}$, with $\Sigma\left|d_{n}\right|^{p}=1$, so that

$$
\begin{aligned}
\left(\sum\left|\hat{f}\left(z_{n}\right)\right|^{q}\right)^{1 / q} & =\sum \hat{f}\left(z_{n}\right) d_{n}=\frac{1}{2 \pi} \int_{-\pi}^{\pi} f \sum d_{n} e^{i z n^{t}} d t \\
& \leq\|f\|_{p}\left\|\sum d_{n} e^{i z n^{t}}\right\|_{q} \leq A\|f\|_{p} .
\end{aligned}
$$

4. Remark. When $\Sigma\left|c_{n}\right|^{2}<\infty$ the series $\Sigma c_{n} e^{i z_{n} t}$ converges in mean square over every interval $(x, x+1)$, uniformly with respect to $x$, and hence represents a function which is almost periodic in the sense of Wiener and Stepanoff. (See [3] and [4].)

Acknowledgement. The author wishes to thank the referee for his simplification of part (ii) of the Theorem.

\section{BIBLIOGRAPHY}

1. A. E. Ingham, Some trigonometrical inequalities with applications to the theory of series, Math. Z. 41 (1936), 367-379.

2. Y. Katznelson, An introduction to harmonic analysis, Wiley, New York, 1968. MR 40 \#1734.

3. R. Paley and N. Wiener, Fourier transforms in the complex domain, Amer. Math. Soc. Colloq. Publ., vol. 19, Amer. Math. Soc., Providence, R. I., 1934. 300-304.

4. E. C. Titchmarsh, A class of trigonometrical series, J. London Math. Soc. 3 (1928), 